\title{
IDENTIDADES ESPAÑOLAS EN AMÉRICA A TRAVÉS DEL ARTE Y LA ARQUITECTURA. ESCENARIOS DE ENTRESIGLOS (1890-1930) Y PROLONGACIONES EN EL TIEMPO
}

\author{
RODRIGO GUTIÉRREZ VIÑUALES \\ Universidad de Granada \\ rgutierr@ugr.es
}

(Recepción: 07/05/2015; Revisión: 21/08/2015; Aceptación: 17/11/2015; Publicación: 02/12/2016)

1. INTRODUCCIÓN.-2. El NEOÁrABE, UN PRIMER ESCENARIO ARQUITECTÓNICO.-3. RECUPERANDO LA ARQUITECTURA COLONIAL. UNA IDENTIDAD HISPÁNICA PARA AMÉRICA.-4. ARTES PLÁSTICAS E HISPANISMO.-5. EPÍLOGO. ESCENARIOS POSTERIORES A 1930.-6. BIBLIOGRAFÍA

\begin{abstract}
RESUMEN
El presente estudio pretende reflejar algunos lineamientos fundamentales en lo que atañe al desarrollo de las artes de cuño español en la América contemporánea, analizando en algunos casos la fortuna historiográfica de ciertas temáticas, y teniendo en cuenta tanto producciones españolas como americanas. Estructuramos el texto a través de una sucesión de escenarios fundacionales en los que se produjeron intercambios de relevancia entre España y América, consolidando una presencia estable y fructífera de la identidad española en aquel continente, con especial atención al primer tercio del siglo XX. Así, centramos el interés en temáticas arquitectónicas como el neoárabe y el neocolonial, el hispanismo y su presencia en las artes plásticas (teniendo en cuenta aquí a la pintura, la escultura y la gráfica), para culminar, a modo de epílogo, con una valoración de los periodos sucesivos, englobando los años de la Guerra Civil, la diáspora española en América, la acción institucional desde la España franquista, hasta llegar a las celebraciones del Quinto Centenario en 1992 y sus reminiscencias, hasta la actualidad.
\end{abstract}

Palabras clave: España; América; arte; arquitectura; identidad. 


\title{
SPANISH IDENTITIES IN AMERICA THROUGH ART AND ARCHITECTURE. STAGES BETWEEN TWO CENTURIES (1890-1930) AND EXTENSIONS IN TIME
}

\begin{abstract}
this study aims to reflect some fundamental guidelines with regard to the development of the arts of Spanish minted in contemporary America, analyzing in some cases historiographical fortune of certain themes, and taking into account both Spanish and American productions. We structure the text through a succession of foundational stages in which relevant exchanges between Spain and America were produced, consolidating a stable and fruitful presence of Spanish identity in that continent, with special attention to the first third of the twentieth century. So, focus interest in architectural themes such as neo-arab and neocolonial, hispanism and its presence in the arts (given here painting, sculpture and graphics), culminating, as an epilogue, with a valuation of successive periods, encompassing the years of civil war, the spanish emigration in America, institutional action from Franco's Spain, reaching Fifth Centenary celebrations in 1992 and his reminiscences, until today.
\end{abstract}

Keywords: Spain; America; art; architecture; identity.

$$
* * *
$$

\section{INTRODUCCIÓN}

En el presente ensayo, dados los lineamientos de la revista Historia y Política en cuanto a establecer ideas, reflejar procesos y significar a los protagonistas, nos pusimos como objetivo principal establecer algunos lineamientos fundamentales en lo que hace a la identidad española en la América contemporánea en cuanto al desarrollo de las artes y, derivado de ello, analizar en algunos casos la fortuna historiográfica de ciertas temáticas, teniendo en cuenta tanto producciones españolas como americanas.

Para llevar a cabo nuestro cometido, estructuraremos el estudio a través de una sucesión de asuntos o espacios que hemos dado por denominar «escenarios fundacionales», en los que se produjeron intercambios de relevancia entre España y América, consolidando una presencia estable y fructífera de «lo español» en aquel continente, con atención al primer tercio del siglo Xx. Se tendrán asimismo en cuenta, en algunos casos, las reciprocidades, es decir, la recepción de «lo americano» en España, o lo que es lo mismo, el papel desempeñado por España para la difusión del arte americano y, en casos, el entendimiento de ello como parte de la propia identidad peninsular. 


\section{EL NEOÁRABE, UN PRIMER ESCENARIO ARQUITECTÓNICO}

Desde la celebración de la primera exposición «universal», la de Londres en 1851, la arquitectura desempeñó un papel fundamental en la consolidación de imaginarios exóticos, pero a la vez, y conforme fueron avanzando las décadas, en el establecimiento de señales identitarias para los países concurrentes. En efecto, el inicio de dichos eventos coincidió con un creciente interés por el rescate y reinterpretación de «estilos históricos». Así, se multiplicaron edificios neoegipcios (muy habituales en cementerios, por el vínculo con la muerte), neorrománicos o neogóticos, entre otras muchas propuestas. Justamente los ámbitos de las exposiciones internacionales fueron muy propicios para divulgar este tipo de testimonios, en tanto se trataba, por lo general, de edificios de carácter efímero. Al ser arquitecturas de corte lúdico, pensadas para eventos masivos y sin condicionantes más allá de los propios eventos, estos fungieron como verdaderos laboratorios de prueba, en los que podían experimentarse estas edificaciones tan peculiares, pudiéndose analizar a posteriori si eran viables o no para insertarse en las propias ciudades de una manera estable.

En este espectro, y durante aquella exposición inicial en Londres, se construyó el paradigmático Crystal Palace, en el cual se insertarían elementos decorativos tomados de la Alhambra. Owen Jones había realizado viajes a Granada, y publicado su Plans, Elevations and Details of the Alhambra (1835-1845) que culminaría en Grammar of ornament (1856), una suerte de manual de diseños tomados del paradigmático edificio, con una doble finalidad, tanto la arqueologista (rescatar detalladamente esos elementos), como la de convertirse en un basamento para aplicaciones a la arquitectura moderna. Este último proceso habría de desarrollarse con gran libertad, no sujetándose los arquitectos y diseñadores al original sino transformándolo, añadiendo otros ornamentos, aplicándole colorido, combinándolos con recursos provenientes de otros cánones estilísticos. Ese «carnaval de estilos» daría pie a lo que se conocería con el nombre de eclecticismo, propio de la segunda mitad del XIX y consagrado por la parisina École des Beaux Arts, referente hasta entrada la siguiente centuria.

Estas consideraciones introductorias son necesarias para entender una de las primeras apropiaciones españolas en el camino de crear una imagen identitaria de carácter internacional. Si bien el neoárabe, como estudió Tonia Raquejo (1989), tuvo mayor aceptación en las islas británicas y por traslación en los Estados Unidos, España la adoptaría como imagen propia. La arquitectura en las exposiciones internacionales fueron convirtiéndose en encuentros en donde no solamente se ponían en evidencia los adelantos comerciales o culturales de los países, sino también una oportunidad de identificarse, de diferenciarse del país de al lado; la arquitectura jugaría un papel trascendental a través de los pabellones nacionales, dado que además esa primera imagen alcanzaba gran valor. España advirtió pronto que un elemento que la distinguía de sus pares europeas era el pasado islámico, y adoptó para varios de sus pabellones el 
neoárabe, que además cumplía con aquel requisito de arquitectura exótica tan caro al clima de las exposiciones. Debemos hacer aquí un pequeño ajuste, mencionando no solamente la arquitectura neoárabe sino también la neomudéjar; la diferencia genérica entre ambas radicaría en que, mientras la primera se caracterizaba por el pastiche, la segunda, además de tener basamentos propiamente españoles, era más mesurada y tenía como uno de sus elementos definidores el uso del ladrillo, que por caso fue habitual en plazas de toros a partir de la que se inauguraría en Madrid en 1874, obra de los arquitectos Lorenzo Álvarez Capra y Emilio Rodríguez Ayuso.

No haremos aquí un recuento del amplio número de pabellones españoles que llevaron el sello alhambresco, y remitimos en tal sentido a los trabajos de María José Bueno (1987) y Daniel Canogar (2000), no sin antes aludir al hastío que provocaría finalmente la repetición del modelo; para la exposición de París de 1900 España decidirá concurrir con un pabellón neoplateresco, diseñado por el arquitecto José Urioste, en el afán de desprenderse del ya estigmático neoárabe. Ni así pudieron contravenir una senda que ya se había internacionalizado: los franceses hicieron construir como una de las atracciones especiales de aquella muestra un espacio que denominaron «Andalucía en tiempo de los moros», dotado de patios alhambrescos en los que interactuaba gente contratada especialmente en el norte de África, integrándose a la vez una Giralda a tamaño natural a la que se podía subir en burro, y hasta una recreación de calles toledanas insertadas, claro está, en Andalucía, como para aumentar aun más la confusión. En eventos posteriores, como en la exposición de Bruselas de 1910, se retornaría no sin cierta resignación, a las pautas neoárabes.

En este proceso de arquitectura identitaria pronto habría de surgir otro tipo de edificios, estos sí a construirse con carácter permanente en las ciudades, como fueron los de las colectividades españolas en América, tanto los representativos de la nación como la de las regionales. Acorde con el carácter de laboratorio arquitectónico de aquel continente, de lugar de pruebas, en ellas prevalecería el sello neoárabe en consonancia con los habituales pabellones de las exposiciones internacionales. En 1904 el arquitecto Miguel Retornano construía el Club Español de Iquique, en el norte de Chile, con colorida decoración interior de raíz alhambrista, a la que se integraba una serie de cuadros sobre el Quijote, inspirados seguramente en los del malagueño José Moreno Carbonero. En 1912 Enrique Folkers, al diseñar el Club Español de Buenos Aires, construía en el sótano un «salón Alhambra» que se utilizaría para celebraciones, insertando, dentro de un cargado ámbito de coloridas yeserías neonazaríes, una gran pintura que ocupaba las cuatro paredes y que recreaba una vista de Granada desde el mirador de San Nicolás, de $360^{\circ}$.

En los años 20 las referencias se multiplicarían. El cine de Hollywood, con películas como la de El ladrón de Bagdad (1924), daría nuevos impulsos a este tipo de manifestaciones, no solo en los Estados Unidos sino también en otros países del continente, potenciados por la fascinación que provocaban las 
exuberantes escenografías. Podríamos en ese sentido hacer referencia a numerosos edificios, anteriores y posteriores a este momento, sobre los que hemos discurrido ya en otros estudios (1) y por lo tanto no enumeraremos aquí. Sí diremos que el pasado islámico seguirá siendo referente para la imagen de España en América, como puede apreciarse en la Casa de España diseñada por Pedro A. de Castro para San Juan de Puerto Rico, en la que se incorpora como elemento singularizado a un costado de la misma, una réplica de la fuente de los leones de la Alhambra, elemento que, a partir de la que en 1862 había mandado hacer el arquitecto Manuel Aldunate para la residencia de la familia Vicuña en Santiago de Chile, habría de repetirse en versiones «cultas» y también populares, a lo largo y ancho del continente.

Las celebraciones de los centenarios u otras conmemoraciones traerían aparejados homenajes de las colectividades españolas en los países americanos, generalmente a través de la donación de un monumento público. Justamente queremos señalar aquí dos: el «arco morisco» que los españoles afincados en el Perú donaron a este país con motivo del Centenario de la independencia en 1921, y el que la colectividad española de Tandil (provincia de Buenos Aires, Argentina) haría construir en aquella ciudad al año siguiente. Respecto del primero, tuvo carácter efímero, ya que, instalado en la Avenida Leguía (hoy Alameda), habría de ser demolido en 1939. El «arco morisco» fue reconstruido en 2002 en el Parque de la Amistad.

\section{RECUPERANDO LA ARQUITECTURA COLONIAL. UNA IDENTIDAD HISPÁNICA PARA AMÉRICA}

Mientras el neoárabe iba irradiándose desde las exposiciones universales, a través de las publicaciones conmemorativas como asimismo gracias a la praxis de arquitectos que asistían a esos eventos, o mecenas que, empalagados con los exotismos, pasarían luego a convertirse en comitentes de edificios similares en sus sitios de residencia, otras variables historicistas, por diferentes circunstancias, iban sedimentando en tierras americanas. Si para el neoárabe el modelo de difusión era el comentado líneas arriba, añadido a la circulación de sus imaginarios desde Inglaterra a Estados Unidos y desde allí a la región caribeña, y luego al resto del continente, fortuna mayor, por algunas sendas comunes, alcanzarían las reinterpretaciones de las arquitecturas de raíz colonial. Al igual que con los otros historicismos, papel fundamental lo jugará la divulgación de libros y revistas de arquitectura desde el país del norte hacia el sur, o la asistencia de arquitectos latinoamericanos, sobre todo cubanos y puertorriqueños, a las universidades estadounidenses. Fue el caso, por ejemplo, del ya citado Pedro A. de Castro.

(1) GutiéRREZ ViÑUALES (2006): 231-259; (2008): 95-122; (2010): 285-307. 
A mediados del siglo XIX se había producido un hecho decisivo como fue la guerra mexicano-estadounidense de 1846-1848 con la que se inició un proceso de adueñamiento por parte de Estados Unidos de las misiones californianas a las que luego se añadiría un proceso de rescate patrimonial en el que cabría la acción de numerosos arquitectos e historiadores. Dicho proceso comenzará a darse con fuerza a partir de la adquisición por parte del Estado de Texas de «El Álamo» en 1883, y la restauración, al año siguiente, de la misión de San Carlos Borromeo del Carmelo; todo ello potenció el entusiasmo por la recuperación de los edificios misionales, buena parte en ruinas (2). La publicación de la novela Ramona (1884), de Helen Hunt Jackson, escenificada en las antiguas misiones, marcaría un boom editorial que acrecentaría la validez de todo el proceso.

Ello derivaría en la invención de un «estilo misionero» (mission style), enmarcado dentro de los historicismos ya reseñados, que, al decir de Susana Torre, se trataría de «la apropiación de los vencedores de las formas urbanísticas y arquitectónicas de los vencidos, pero vaciados de los significados sociales que les dieron origen» (3). Así, surgirían edificaciones como las de la Universidad Stanford en Palo Alto (1885) de Charles Coolidge Allerton, algunas inspiradas en edificios de adobe de los pueblos de indios; el pabellón de California para la exposición universal de Chicago en 1893, obra de Arthur Page Brown; la casa «El Alisal» (1897-1910), de Charles Lummis, fotógrafo, periodista y promotor de las arquitecturas californianas nativas y en especial las misioneras, que además propulsaba una suerte de retorno espiritual al estilo de vida de la época colonial española. A todo ello hay que agregar la tarea de la arquitecta Julia Morgan vinculada a la familia de magnates Hearst, en obras como la terminación de la mansión familiar «Hacienda del Pozo de Verona» en Pleasanton o el edificio del periódico Los Ángeles Examiner entre 1913 y 1915, ya en plena fase de agotamiento de los postulados del mission style (4).

La pasión por «lo español» derivó también en un proceso de expolio de mobiliario original, evitando las réplicas, que se veía como necesario para dotar de autenticidad a este tipo de edificios, dado en especial en las primeras décadas del siglo Xx: «miles de objetos de arquitectura fueron extraídos de las obras renacentistas, manieristas y barrocas españolas. Desde claustros enteros que se llevaron desarmados para montarlos en Estados Unidos hasta puertas, rejas, cielorrasos, torres, paneles de azulejos, etc. que se incorporaron a museos públicos y privados y a muchas residencias particulares» (5). En este escenario, justamente William Randolph Hearst, personaje que inspiraría a Orson Wells su Ciudadano Kane (1941), se convirtió en el mayor comprador de arte español, de manera compulsiva y sin reparar en turbiedades para conseguir sus objeti-

(2) GELLNER (2002): 8-10.

(3) TORRE (1994): 48.

(4) GutiÉRREZ ViÑUALES (2014a).

(5) GutiéRREZ Y TARTARINI (1997): 66. 
vos (6). En Nueva York, otro millonario movido por una acendrada pasión por lo español, Archer M. Huntington, fundaba en 1904 la Hispanic Society de Nueva York, conformando una notable biblioteca de temas hispánicos y un museo de arte.

Pero volviendo a Hearst, de las obras comisionadas a Julia Morgan habría de sobresalir «La Casa Encantada», más conocida como Hearst Castle (19191947), ubicada en San Simeón, y en la que se ve ya la prédica del estilo «renacimiento español», creciente desde la exposición de San Diego de 1915. Muy pronto se disparó el desarrollo urbano en zonas residenciales y vacacionales, y el furor por estas formas derivó en una excitación constructiva en la que se aplicaron estéticas populares (misioneras y andaluzas) y norteafricanas. Se concretó una verdadera «fiebre hispana», que dejó, sobre todo a California y a Florida, con varias poblaciones españolas.

Las alternativas arquitectónicas vinculadas a ese «estilo californiano» (7), de fuerte impronta fachadista, suelen dividirse en dos: una, la ya referida versión popular conocida como mission style, de notoria fortuna en los últimos lustros del siglo XIX e inspirada en la arquitectura de las veintiún misiones californianas; dos, la conocida como «renacimiento español», que toma como referencia el barroco español (realmente el mexicano) con sus cargas decorativas, que tendría su punto culminante en la Exposición Panamá-California celebrada en San Diego en 1915, en las arquitecturas del Balboa Park. Ambas vertientes están caracterizadas por el eclecticismo, en tanto formaron parte de ellas, combinados, otros testimonios como la arquitectura de los indios Pueblo, rasgos victorianos, mediterraneístas italianos, neoárabes o neomayas (8), entre otros aspectos (9). El éxito de estas construcciones se dio fundamentalmente en California y Florida, zonas ambas de notable influjo turístico, y en especial en las décadas de 1920 y 1930 (10).

Respecto de la exposición de San Diego, el arquitecto seleccionado para el evento fue Bertram Grosvenor Goodhue, quien tenía como antecedente una serie de viajes a México a partir de inicios de 1892 (11) y su trabajo como diseñador de planos para los tomos que Sylvester Baxter publicaría bajo el título Spanish Colonial Architecture in Mexico, a partir de 1901. Estos precedentes serán

(6) Merino de CÁCERES y Martínez Ruiz (2012).

(7) MCMiLLIAN (2002).

(8) INGLE (1984).

(9) Dada esta heterogeneidad, autores como D. J. Waldie mencionaban como dos fases del proceso, por un lado el Mission style, pero por otro el California style, capaz de contener en sí al «renacimiento español» y a las otras vertientes «mediterráneas», desarrolladas sobre todo en los años 20. Cfr.: KEATON y WALDIE (2007). El término California style comenzará a utilizarse a finales de esa década, englobando a los términos Mission, Spanish o Mediterranean, habituales hasta entonces. Cfr.: MCMillian (1996): 18.

(10) Con extensiones directas hacia México. Recomendamos a los efectos PiÑERA RAMírEZ y BEJARANO SuÁREZ (2011): 159-184.

(11) Algunas de estas experiencias, y una serie de dibujos, los recogió en GoodHUE (1892). 
claves para que Goodhue determine como estética predominante de las construcciones en el Parque Balboa (12), sede del evento, lo que se dará por llamar «renacimiento español», basado en una mirada ecléctica sobre el barroco mexicano y peninsular, integrando arcadas, porches, balcones, ventanas con celosías, rejas de hierro forjado, toldos de lona, patios con fuentes y abundante uso de la azulejería sevillana importada o de inspiración hispalense (13). Estas edificaciones enlazaban con una serie de obras ejecutadas en la década de 1880 en Florida, en las que se mezclaban rasgos españoles y mediterráneos, e inclusive notas moriscas. En los años siguientes a este evento, la publicación de libros como The Franciscan Mission Architecture of Alta California (1916) de Rexford Newcomb, o Spanish homes of California (14), potenciarían el que muchos arquitectos de todo el continente americano se lanzaran a construir bajo patrones estilísticos «hispánicos», concientizando acerca de las identidades pretéritas. A ello se sumaría, aquí también, la industria cinematográfica hollywoodiense, con películas que incluían escenografías con arquitecturas neocoloniales (15).

Justamente, este término de neocolonial, es el que se impondría en Latinoamérica para las arquitecturas de raíz hispana, en medio de un proceso identitario potenciado desde los círculos intelectuales, y del que tomaron parte activa arquitectos, artistas y literatos. Esta realidad sería estimulada por la celebración de los centenarios, en tanto momento de balance y debate sobre proyecciones futuras de los países, y, poco tiempo después, por el estallido de la primera guerra mundial, en el que el modelo cultural europeo, hasta entonces casi indiscutible, desciende en su consideración y propicia una mirada introspectiva americanista. Si a ello sumamos los efluvios arquitectónicos «hispanistas» arribados desde Estados Unidos a través de revistas de aquellas latitudes y otras propias que incorporaban información del país del norte, «lo español» pasaba a conformar parte singular de la identidad americana. Dicho de otra manera, la identidad española se arraigaba como componente de las esencias del continente.

No obstante, lo hispánico (16) fue incorporándose de forma gradual y no sin conflictos y ciertos recelos. Nombres como los de Federico Mariscal y Manuel Jesús de Acevedo en México, Martín Noel y Ángel Guido en Argentina, Héctor Velarde en Perú, Roberto Dávila Carson en Chile, o Evelio Govantes y Félix Cabarrocas en Cuba plantearon en sus arquitecturas adaptaciones de modelos de raíz colonial, en consonancia con las pautas historicistas propulsadas desde la École des Beaux Arts. Pero lo hacían con la dimensión ideológica de situarse dentro de corrientes nacionalistas y americanistas, como lo serían también la

(12) Una síntesis con muy buen repertorio fotográfico puede verse en HUDSON (2000).

(13) Del material consultado acerca de este tipo de construcciones en el primer tercio del siglo XX, destaca por textos e imágenes el ya citado GELLNER (2002). Incluye asimismo ejemplos más recientes.

(14) Spanish homes...(1925).

(15) GUTIÉRREZ ViÑUALES (2014a).

(16) GUTIÉRREZ ViÑUALES (2003a): 167-185. 
arquitectura neoprehispánica, y, en cuestiones pictóricas, el paisaje, las costumbres y el indianismo/indigenismo.

Si tomamos como emblemático el caso mexicano, y como hito a la conferencia pronunciada por el citado Mariscal en 1915 sobre La Patria y la arquitectura nacional, debemos señalar como antecedentes algunas operaciones previas, y en especial dos, una externa ya citada, la realización de la obra Spanish Colonial Architecture in Mexico de Sylvester Baxter en 1901, y una interna, el encargo de la Dirección General de Monumentos al fotógrafo Guillermo Kahlo para documentar gráficamente las iglesias coloniales del país. El papel de la fotografía en la divulgación y puesta en valor del legado hispánico sería esencial en otros países del continente como demostraron las fotos de Martín Chambi en la región del Cuzco, los hermanos Vargas en Arequipa, Manuel Mancilla en la región altoperuana o el italiano Luis D. Gismondi en La Paz, reproducidas en tarjetas postales, publicaciones periódicas y libros, entre estos los de varios arquitectos e historiadores del arte dedicados a analizar y teorizar sobre el pasado colonial, de lo que hablaremos más adelante.

Volviendo a México, la revalorización del pasado colonial, y la incorporación de «lo español» como sustancia de un nuevo nacionalismo, devino en ordenanzas como la del gobierno de Venustiano Carranza de eximir de impuestos a quienes construyeran en «estilo colonial». Más aun, encontraría un ferviente defensor en el secretario de Instrucción Pública José Vasconcelos, promotor del movimiento muralista, de fuerte sesgo indigenista, en los años 20 , pero «neocolonialista» en cuestiones arquitectónicas, como pudo comprobarse en el estímulo de ejemplos paradigmáticos de la talla del pabellón mexicano para el centenario brasileño (Río de Janeiro, 1922), diseñado con fachada retablo por el arquitecto Carlos Obregón Santacilia (17).

La «Hispanofilia» (18) se extendería asimismo por la región caribeña, afectando sensiblemente a ciudades como San Juan o Ponce en Puerto Rico, a través de la acción de arquitectos como Pedro Adolfo de Castro o Rafael Carmoega, formados en Estados Unidos, o en La Habana, donde el prestigio de Evelio Govantes y Félix Cabarrocas se sustentó en buena medida en la recreación de formas coloniales. Ambos fueron autores del pabellón cubano de la Exposición Iberoamericana de Sevilla en 1929 y, a continuación, del conjunto arquitectónico en Rancho Boyeros que integró al Instituto Técnico Industrial diseñado en estilo neocolonial, residencias de la urbanización Lutgardita con notas neoárabes y el teatro de la misma en lenguaje neomaya, efectivizando de esta manera una praxis polifacética a tono con las corrientes eclecticistas, en momentos en que se iba irradiando el racionalismo y la desornamentación.

En cuanto a Sudamérica, a la práctica arquitectónica acompañó la reflexión teórica y crítica como fue el caso de Héctor Velarde en el Perú, que alternó

(17) CORTINA (1992).

(18) Vivoni Farage y Álvarez Curbelo (1998). 
proyectos neocoloniales con otros de corte racionalista (19). Ricardo Severo, en Brasil, sentó un punto de partida para el neocolonial con una conferencia impartida en 1914 en la Sociedade de Cultura Artística de São Paulo, sobre la arquitectura tradicionalista y la necesidad de mirar al pasado colonial para pensar nuevas arquitecturas. A ello sobrevendrían viajes iniciáticos, de redescubrimiento de las arquitecturas populares y coloniales de la región de Minas Gerais, que enlazó la práctica de su generación con los modernistas de São Paulo desde principios de los años 20 (20). José Marianno Filho sería otro nombre esencial para la imposición de lenguajes neocoloniales, presentes en los pabellones brasileños en las exposiciones de Filadelfia (1925) y Sevilla (1929) (21).

En la Argentina sobresalen los nombres de Ángel Guido y Martín Noel, aunque en cuestiones teóricas debemos señalar como antecedente una conferencia pronunciada en 1913 por el arquitecto Alejandro Christophersen poniendo en evidencia los valores de la arquitectura colonial. Al año siguiente sería Noel quien seguiría esa misma senda a través de una disertación sobre Arquitectura virreinal, centrando especialmente su atención en Perú y Bolivia (22). Noel sería el autor del pabellón argentino de la exposición de Sevilla de 1929, su obra máxima dentro del neocolonial. En cuanto a Guido, publicaría en 1925 su ensayo Fusión Hispano-indígena en la arquitectura colonial, prolegómeno de una de sus obras emblemáticas, la residencia del escritor Ricardo Rojas en 1927, uno de los ideólogos principales de la fusión de estilos en la definición de un arte nacional y americano (23).

En todo este proceso de consolidación de una «identidad española» en América, un punto culminante lo supondrá la Exposición Iberoamericana de Sevilla de 1929, no solamente por sus evidentes significados simbólicos, sino en lo que respecta a las cuestiones arquitectónicas, confluyendo pabellones que supusieron un abanico de ejemplos historicistas de raíz americana: el neoprehispánico, el neocolonial y la fusión de ambas culturas estéticas. El crack de la bolsa neoyorquina, pocos meses después de inaugurado aquel evento, pondría fin al optimismo cultural de los años 20, dando paso a la crisis. El neocolonial comenzaba su declive, aun sin quedar del todo sentenciado, tanto ante los embates del «estilo internacional» como por el alto coste económico que demandaban sus construcciones, ya no tan viables en medio de una crisis, además de la necesidad de una mano de obra particularmente cualificada.

(19) GuTIÉRREZ (2002).

(20) AMARAL (1994)

(21) LEMOS (1994): 159.

(22) Ver KuON ARCE et al. (2009).

(23) GUTIÉRREZ (1978). 


\section{ARTES PLÁSTICAS E HISPANISMO}

Indudablemente, otro factor esencial en este derrotero de conexiones hispanoamericanas lo propiciarán las artes plásticas, principalmente la pintura y la escultura, pero también con mucha fuerza las artes gráficas, que propiciarán la difusión a gran escala de los imaginarios peninsulares. Al margen de Cuba y Puerto Rico que siguieron siendo parte de España hasta finales del XIX, en el resto de los países se fue arraigando de a poco, y hacia esas fechas, por un lado el coleccionismo de arte español, y por otro la contratación de escultores españoles para colaborar con el proceso de monumentalización que aumentaría notablemente conforme se acercaron los centenarios.

Es bien sabido que Cuba, durante el XIX aunque manteniéndose en el XX, consolidó un importante acervo de pintura española, que en especial en las últimas décadas fue objeto de varias exposiciones en España, generales o parciales, con obras pertenecientes al Museo Nacional de Bellas Artes de La Habana (24). Pero también fue destacado el papel del mercado en Argentina principalmente, y en otros países como Chile, Uruguay (25), Perú, México (26) o Estados Unidos, inclusive Brasil, en donde se conformaron importantes colecciones de pintura española. De Estados Unidos hemos de mencionar nuevamente la fundación de la Hispanic Society of America en 1904, por obra y gracia de Archer M. Huntington, quien además de conformar una de las mejores bibliotecas hispanas de la época, hizo lo propio con acervos pictóricos (27); en esta línea lo más relevante habría de ser el encargo realizado a Joaquín Sorolla para pintar las «regiones de España» tarea que demandó la dedicación del artista hasta su muerte en 1923, y que dotó a la institución neoyorquina de uno de los conjuntos más notables de cuantos se hicieron en la época que estudiamos, tarea que le continuaría luego el granadino José María López Mezquita.

Es paradigmático el caso argentino, objeto de numerosas publicaciones en las últimas décadas (28). En 1897, el marchante de cuadros José Artal decidió llevar a Buenos Aires, a sabiendas de la existencia de un incipiente mercado y sobre todo de un alto número de inmigrantes españoles, un conjunto de pinturas recogidas en distintos talleres de artistas en la Península, con la finalidad de tantear aquel ámbito. Los cuadros le fueron arrancados literalmente de las manos: vendió prácticamente todo el conjunto. Esta constatación de un éxito

(24) Destaca entre todas: Crespo LarrazÁbal y CALVo Serraller (1995).

(25) Laroche (1992); Carbajal y Moreno Tonelli (1992).

(26) PÉrez SÁNCHEZ (2000).

(27) En exposiciones realizadas en Madrid: Sorolla y la Hispanic Society. Una visión de la España de entresiglos, Museo Thyssen-Bornemisza, 1998; y De Goya a Zuloaga. La pintura española de los siglos XIX y XX en The Hispanic Society of America, Sala de exposiciones del BBVA, 2000.

(28) Cruz Valdovinos (1991); García-Rama (1994); Santa Ana Álvarez Osorio (1995); FERNÁNDEZ GARCÍA (1997). 
esperado solo a medias, le llevó a concretar ya de una manera periódica este tipo de exposiciones en la capital argentina, casi siempre con la misma fortuna, lo que llevó a otros comerciantes como el andaluz José Pinelo a seguir el mismo camino.

Las colecciones privadas (sobre todo) y públicas argentinas se nutrieron en muy poco tiempo de ingentes cantidades de pintura española. Las temáticas eran las habituales del regionalismo español y por ende las más requeridas por los coleccionistas por su carácter de «españolas»: majas, gitanos y costumbres en general, paisajes, asuntos religiosos, en algún caso retratos. Las mismas impactarían en artistas locales y reconocidos como fueron los casos del peruano Teófilo Castillo o el argentino Emilio Caraffa, influidos claramente por el preciosismo del catalán Mariano Fortuny. El argentino Fernando Fader obtendría el premio adquisición del IV Salón Nacional de su país en 1914, con Los mantones de Manila, óleo de clarísimas reminiscencias españolas, con un tema, el de las majas, que sería también abordado por Jorge Bermúdez, Jorge Larco o José Antonio Terry en ese país. Otros artistas como el venezolano Tito Salas, el ecuatoriano Joaquín Pinto o los mexicanos Diego Rivera y Ángel Zárraga, por citar solamente un puñado de nombres notables dentro de una lista muy extensa, dedicaron parte de su producción a temáticas costumbristas y paisajísticas españolas. También lo harían nombres de las vanguardias latinoamericanas como el cubano Wifredo Lam, los peruanos Carlos Quízpez Asín o Manuel Pantigoso, la brasileña Tarsila do Amaral, los uruguayos Carlos Alberto Castellanos y Rafael Barradas, o los argentinos Norah Borges y Antonio Berni.

Pero nos hemos adelantado en el tiempo. Decíamos que las obras procedían de los atelieres de artistas, que por lo general acostumbraron a enviar no sus obras principales sino las de segunda línea o a veces hasta los bocetos de sus grandes composiciones, todo ello adquirido sin rechistar por los coleccionistas. Al llevarse a cabo en Buenos Aires la Exposición Internacional del Centenario en 1910, y al contemplar en la sección española los lienzos de Ignacio Zuloaga, Hermen Anglada Camarasa y otras primeras espadas de la pintura española del momento, aquellos comprendieron que hasta ese momento habían estado comprando obras de segunda y tercera categoría. Esto incidió para que a partir de entonces el nivel de exigencia aumentara notablemente y ya no fue tan habitual el ingreso de aquellas obras menores. El influjo de los dos artistas mencionados sería notorio: Zuloaga tendría seguidores estéticos como los argentinos Jorge Bermúdez y José Antonio Terry, o el peruano José Sabogal, curiosamente todos coincidentes en Tilcara (Jujuy) a finales de la segunda década de siglo desarrollando una pintura de tipos costumbristas que derivaría, para el caso de Sabogal, en la pronta instalación de la escuela indigenista en el Perú (29). En cuanto a Anglada, el influjo va a ser directo en una legión de artistas latinoamericanos, sobre todo argentinos, que le siguieron tras el Centenario

(29) GUTIÉRREZ ViÑUALES (2000): 396-412. 
en sus talleres de París y Pollensa (Mallorca), temas que hemos estudiado en otras ocasiones (30).

En el sur, otro país en el que se formaron buenas colecciones de pintura española fue Chile, tanto con obras que pasaron a acervos públicos como el Museo Nacional de Bellas Artes (31), como las que integraron colecciones privadas, por caso las que se pueden ver hoy en la Quinta Vergara de Viña del Mar, propiedad de los Errázuriz, cuyo palacio en Buenos Aires también cuenta con un importante conjunto, entre ellos magníficos retratos de Joaquín Sorolla. Para el caso chileno fue decisiva la llegada, en 1908 y hasta 1915, del pintor gallego Fernando Álvarez de Sotomayor, en calidad de profesor de la Academia, tanto para la difusión de la pintura regionalista española como para determinarla como estética de formación en esa institución. Discípulos suyos en Santiago de Chile como Alfredo Helsby o Arturo Gordon, entre muchos otros, en cierta medida fueron durante un tiempo una suerte de regionalistas españoles pero situados en Chile. Años después de su regreso, Sotomayor ingresaría a la Real Academia de Bellas Artes de San Fernando con un discurso sobre «Nuestras relaciones artísticas con América» (32).

Es necesario señalar estos escenarios donde no solamente la pintura española ejerció influjo a través de obras llegadas desde la Península, sino también el hecho de que se radicasen en América artistas españoles con buenas aptitudes y cierto reconocimiento como el señalado caso de Sotomayor. En la Argentina podríamos mencionar a los valencianos Julio Vila y Prades y Ernesto Valls, dos de los discípulos más notables de Sorolla, al gibraltareño Gustavo Bacarisas, el cantillanero Ricardo López Cabrera o el granadino José de Larrocha, todos ellos moviéndose entre las estéticas aprehendidas en España pero alternando temáticas caras a una u otra orilla (33). En México debemos considerar la figura de Juan Téllez, como en Brasil las de Modesto Brocos o Agustín Salinas y Teruel entre muchos otros (34). Por lo general estos actuaron en los países de destino como «artistas españoles»; otros muchos, de origen peninsular, terminaron por meterse de lleno en las dinámicas de esas naciones de acogida, tanto que al final su consideración se dio más dentro de esos medios sin a veces llegar a conocerse (ni a posteriori rescatarse) su obra en España. El caso de Argentina vuelve a ser en este sentido emblemático: es el caso de prolíficos ilustradores de revistas y libros en el país como los gallegos José María Cao y Juan Carlos Alonso, el andaluz Manuel Mayol, el asturiano Alejandro Sirio o los catalanes Luis Macaya, Pompeyo Audivert, José Planas Casas, Francisco Fábregas o Andrés Dameson

(30) GutiérReZ Viñuales (2001): 189-203. Ver también Miralles y SanjuÁn (2003), y LLADÓ i POL (2006).

(31) PÉREZ SÁNCHEZ (1999).

(32) ZAMORANO PÉREZ (1994).

(33) GUTIÉRREZ ViÑUALES (2003a): 167-185; y (2003b).

(34) TeiXeira Leite (1996). 
entre muchos otros (35). Todos antes de 1930. En Uruguay es destacada la figura del gallego Melchor Méndez Magariños, que xilografió numerosas obras de la vanguardia literaria de los años 20 (36).

Otro escenario de inserción española en América durante el primer tercio del siglo XX será el de la escultura, y en especial debemos referir a los testimonios vinculados al proceso de monumentalización de los espacios públicos americanos que estudiamos de manera amplia en 2004 (37). Los nombres vinculados a este escenario son varios, aunque sobresaliendo sobre todos ellos los de los catalanes Agustín Querol y Miguel Blay, y el valenciano Mariano Benlliure, receptores de numerosos encargos y artífices de notables obras como el Monumento de los españoles en Buenos Aires (1909-1927) por parte de Querol, el monumento a San Martín (1921) de Benlliure para el centenario peruano en Lima, o la estatua de Vasco Núñez de Balboa en Panamá (1924), obra compartida por Benlliure y Blay. Mención aparte merece la instauración, en muchas ciudades del continente de las «plazas España», dotadas con estatuaria alusiva, en donde figuras alegóricas, de reyes, descubridores y conquistadores, o personas de la cultura (Miguel de Cervantes en este sentido representa la quintaesencia de aquella), son habituales.

El periodo está marcado por el flujo de intelectuales que se desplazan desde y hacia uno y otro lado del Atlántico; así pasan por América, entre muchos otros, Vicente Blasco Ibáñez, José Ortega y Gasset, Ramón Menéndez Pidal o Eugenio D'Ors. La presencia de España en las exposiciones del Centenario será nutrida en todas las ramas de la producción y el saber, no solamente en la faz artística tal como hemos reseñado. España devolvería gentilezas en la tan postergada Exposición Iberoamericana, concretada finalmente en Sevilla en 1929, donde el despliegue de la arquitectura y las artes será notable desde los países americanos. Arquitectos de prestigio reconocido diseñarán pabellones para sus países, como es el caso de los cubanos Evelio Govantes y Félix Cabarrocas, el mexicano Manuel Amábilis, el argentino Martín Noel, el uruguayo Mauricio Cravotto o el chileno Juan Martínez Gutiérrez (38). En esos espacios se celebrarán amplias exposiciones artísticas como las de las mexicanas escuelas de pintura al aire libre fundadas por Alfredo Ramos Martínez, se ejecutarán murales como los de Alfredo Guido y Gustavo Bacarisas para el pabellón argentino o los de Arturo Gordon para el chileno, o se ejecutarán nutridas decoraciones como las creaciones escultóricas de Rómulo Rozo para el colombiano o las pinturas de Manuel Domingo Pantigoso para el peruano. La Exposición de Sevilla marcará un punto culminante a la vez que un canto del cisne para un periodo notable de las relaciones entre España y América, el del reencuentro cultural tras el siglo de distanciamiento

(35) GuTIÉRREZ ViÑUALES (2014b).

(36) GUTIÉRREZ ViÑUALES (2014c): 73-84.

(37) GutiérReZ Viñuales (2004).

(38) GRACIANI GaRCÍA (2010). 
que sucedió al proceso emancipatorio. Desde entonces, con mayor fuerza que antes, España hallaría en América elementos reconocibles de su cultura, adoptados y reelaborados en aquel continente.

\section{EPÍLOGO. ESCENARIOS POSTERIORES A 1930}

En nuestro itinerario, el año 1929 determina dos sucesos inconexos pero decisivos para el derrotero del arte y la identidad española en América: por un lado la citada Exposición Iberoamericana de Sevilla, y por otro, el crack de la bolsa de Nueva York con sus consecuencias para la economía global y en especial para este escenario cultural que veníamos tratando. De pronto el ambiente se enturbia, las naciones tienden a encerrarse en sí mismas, a apartarse del clima optimista de los 20, y, en cuestiones artísticas, haciendo prevalecer manifestaciones de mayor compromiso social. España transitaría hacia la Guerra Civil de 1936, que en los países americanos se viviría muy de cerca: creadores como el ecuatoriano Camilo Egas, el mexicano David Alfaro Siqueiros o los argentinos Raquel Forner, Antonio Berni, Demetrio Urruchúa, Manuel Kantor, Aquiles Badi o César López Claro, entre otros, plasmarían en sus obras la crudeza de la contienda, desde visiones plenamente realistas, o valiéndose de sendas simbólicas en muchos casos surrealizantes.

En el año del fin de la Guerra Civil, que fue la del inicio de la segunda guerra, en la Feria Internacional de Nueva York se producía la exhibición del Guernica de Picasso, pintado dos años antes en París con motivo de otra exposición internacional, trasladándose desde allí a la ciudad estadounidense. La estancia en Nueva York de esa obra hasta su recuperación por parte de España en 1980, supondría la ubicación de la misma, de una manera decisiva, en las historias del arte que se estandarizarán a lo largo de la centuria. Más allá de ello, sería en la feria de 1939 cuando la verían directamente numerosos artistas americanos que confluyeron allí por lo general para trabajar en los pabellones de sus países; en muchos de ellos el Guernica dejará una huella indeleble, siendo evidente en la serie de temas bíblicos pintada por el brasileño Cándido Portinari que se encuentran hoy en el MASP de São Paulo (39), pero también en las obras del mexicano Rufino Tamayo o el cubano Lam. Bien es verdad el Guernica, aun siendo española su temática, no puede entenderse como «hispanista» desde un punto de vista estético.

Otro escenario, que ha tenido en los últimos años una revalorización en cuanto a su estudio, es el proceso de diáspora española hacia América que podríamos situar temporalmente desde el estallido de la Guerra Civil hasta la apertura franquista de finales de los 50 e inicios de los 60. Al decir diáspora incluimos tanto los artistas del exilio como aquellos que se desplazaron por

(39) MagalhãES (1999): 41-43. 
propia voluntad, sin verse obligados a la huida. No es menester elaborar aquí un listado, lo que sería interminable (inclusive si decidiéramos incluir únicamente a los «importantes»), pero podríamos saltar de geografía en geografía hallando arquitectos como Félix Candela Outeriño en México, Antonio Rodríguez Orgaz en Colombia o Antonio Bonet Castellana en el ámbito rioplatense; escultores como José de Creeft en Estados Unidos, Ángel Botello en Puerto Rico, Victorio Macho en Perú, Pablo Serrano en Uruguay o Jorge Oteiza en Colombia; pintores de la talla de Luis Quintanilla, José Guerrero y Esteban Vicente en Estados Unidos, Antonio Rodríguez Luna, José Moreno Villa o José Renau en México, Eugenio Granell en Santo Domingo, José Balmes en Chile, o Luis Seoane, Manuel Colmeiro, Maruja Mallo o Manuel Ángeles Ortiz en la Argentina; fotógrafos como Kati Horna en México o José Suárez en la Argentina; críticos de arte como Francisco Gil Tovar en Colombia, Antonio Romera y Leopoldo Castedo en Chile, o Arturo Serrano Plaja en Argentina. En gran medida, la labor de estos artistas (40) estuvo vinculada a numerosos emprendimientos editoriales por parte de españoles emigrantes (mayoritariamente exiliados en este caso), en órganos como las revistas Romance en México, o Correo Literario, De mar a mar, Cabalgata o Saber vivir en Argentina.

La tarea de la mayor parte de ellos se dio dentro de un clima más internacional, con lo cual la impronta de las estéticas «españolas» fue diluyéndose. Los emigrantes supieron integrarse a los ámbitos artísticos receptores y, en cierta medida, se convirtieron en artistas americanos, aun cuando en muchos casos continuaron acudiendo a simbologías hispanas como las obras sobre el éxodo que pinta Rodríguez Luna en México, o las continuas referencias a lo gallego que plasma Seoane en su obra argentina. Podemos hablar, pues, parafraseando a Ticio Escobar, de «identidades en tránsito». Habría que determinar la pertinencia y el interés que pudiera tener, en la obra de todos ellos, qué de «español» legaron a sus países de destino, pero más aun, entender los procesos artísticos propios de esos países para entender esa obra en toda su dimensión. En definitiva, que los españoles allí terminaron, por lo general, siendo más americanos que españoles.

Mientras esto sucedía en la diáspora, en España, culminada la Guerra Civil, el gobierno franquista comienza a aplicar políticas culturales hacia América, de una manera decidida: en 1941 se funda en Madrid el Museo de América, en 1946 el Instituto de Cultura Hispánica (41), en 1947 itinera por diversos países americanos una magna exposición de «Arte español contemporáneo» organizada desde el Estado en donde se recurre en la mayor parte de sus contenidos a estéticas periclitadas desde hacía décadas. En 1951 se lleva a cabo en Madrid la Primera Bienal Hispanoamericana (42), de la que se harían dos ediciones más,
(40) BRIHUEGA (2009).
(41) GonZÁlez CASASNOvas (2003).
(42) Cabañas Bravo (1996). 
una en La Habana en 1953 y otra en Barcelona en 1955, abriendo un proceso que en cierta medida tuvo su cierre en la importante muestra «Arte de América y España» exhibida en Madrid y Barcelona en 1963, organizada por el Instituto de Cultura Hispánica y comisariada por Luis González Robles, en las que una incipiente modernidad manifestada ya en la bienal barcelonesa del 55 se consolidaba con una amplio muestrario de obras de carácter abstracto que Franco observó perplejo en la inauguración, como se ve en las filmaciones para el NO-DO. Habrá una continuidad en la erección de estatuas a conquistadores y fundadores españoles en América que tenía antecedentes en las de Francisco Pizarro en Lima (1935) y dos de Sebastián de Belalcázar para Cali y Popayán ejecutadas, también en los 30, por Victorio Macho; la más notoria será la de Pedro de Valdivia en Santiago de Chile (1962) realizada por Enrique Pérez Comendador.

Entre 1945 y 1956 se publicó la Historia del Arte Hispanoamericano en tres tomos, a cargo de los españoles Diego Angulo Iñiguez y Enrique Marco Dorta, y el argentino Mario J. Buschiazzo, una de las obras fundacionales de la historiografía del arte de aquel continente. Fue un basamento sobre el cual se siguieron construyendo (y se continúan) diversos estudios sobre la presencia española en la América virreinal. Si bien a lo largo de este texto, y en especial en las notas, hemos dejado amplias referencias bibliográficas y hemerográficas que sustentan un proceso que advertimos muy amplio y complejo, es menester destacar que la mayor producción se viene dando en los últimos veinticinco años. Si con claridad hemos visto que un primer gran momento de la identidad española en la contemporaneidad americana se centra entre 1898 y 1929 (desde la pérdida de Cuba y Puerto Rico hasta la Exposición Iberoamericana y la crisis económica, con los Centenarios en medio), y otro periodo intenso se sitúa entre 1936 y principios de los 60 (desde la Guerra Civil hasta los primeros años tras el fin de la autarquía), es claro que un tercer momento «cultural» lo representan los años anteriores y posteriores al año 1992, el del llamado «Encuentro», proceso abierto hasta la actualidad en diferentes facetas.

En lo que atañe al arte de ese tercer periodo, además de destacar la realización de importantes muestras como la de Arte en Iberoamérica organizada por la británica Dawn Ades y llevada a cabo en 1989 en el Palacio de Velázquez del Retiro madrileño, o la de Arte Latinoamericano del siglo Xx, comisariada por Waldo Rasmussen y exhibida durante la Expo de Sevilla en el 92 (luego llevada a París y a Estados Unidos), se hace imprescindible el destacar, como hemos hecho en anteriores trabajos (43), el surgimiento de instituciones americanistas como el Centro Atlántico de Arte Moderno en Las Palmas de Gran Canaria en 1989, o la Casa de América en Madrid, el Museo Extremeño e Iberoamericano de Arte Contemporáneo (MEIAC) en Badajoz (44) y el Instituto de América en

(43) Ribeiro dos Santos y GutiérRez Viñuales (2012).

(44) Franco Domínguez (2005). 
Santa Fe de Granada (1992). Otras instituciones serán los museos de los exiliados ubicados en distintas comunidades, y que resguardan la memoria de activos protagonistas del arte español en tierras americanas (45).

\section{BIBLIOGRAFÍA}

Amaral, A. (comp.) (1994). Arquitectura Neocolonial. América Latina, Caribe, Estados Unidos. São Paulo: Fondo de Cultura Económica.

Brihuega, J. (dir.) (2009). Después de la alambrada. El arte español en el exilio (19391960). Madrid: Sociedad Estatal de Conmemoraciones Culturales.

Bueno Fidel, M. J. (1987). Arquitectura y Nacionalismo. Pabellones españoles en las exposiciones universales del siglo XIX. Málaga: Universidad.

Cabañas Bravo, M. (1996). La política artística del franquismo. El hito de la Bienal Hispanoamericana de Arte. Madrid: CSIC.

Canogar, D. (2000). Pabellones españoles en las Exposiciones Universales. Madrid: Sociedad Estatal Hannover 2000-El Viso.

Carbajal, M. y Moreno Tonelli, J. (1992). La Influencia española en las artes visuales del Uruguay. Montevideo: Ediciones Galería Latina.

Cortina, L. (dir.) (1992). El Neobarroco en la Ciudad de México. México: Consejo Nacional para la Cultura y las Artes.

Crespo Larrazábal, M. y Calvo Serraller, F. (1995). Pintura Española del siglo XIX del Museo de Bellas Artes de La Habana. Madrid: Fundación Cultural Mapfre Vida.

Cruz Valdovinos, J. M. (coord.) (1991). 120 años de pintura española. Buenos Aires: Museo Nacional de Bellas Artes.

Fernández García, A. M. (1997). Arte y emigración. La pintura española en Buenos Aires, 1880-1930. 2 vols. Oviedo: Universidad.

Franco Domínguez, A. (ed.) (2005). En las fronteras / In borderlines. Arte latinoamericano en la colección del MEAIC. Badajoz: Museo Extremeño e Iberoamericano de Arte Contemporáneo.

García-Rama, R. (dir.) (1994). Otros emigrantes. Madrid: Caja Madrid.

Gellner, A. (2002). Red tyle style. America's spanish revival architecture. New York: Viking Studio.

González Casasnovas, I. (coord.) (2003). La huella editorial del Instituto de Cultura Hispánica. Madrid: Agencia Española de Cooperación Internacional.

Goodhue, B. G. (1892). Mexican memories. New York: G. M. Allen Co.

Graciani García, A. (2010). La participación internacional y colonial en la Exposición Iberoamericana de Sevilla de 1929. Sevilla: Universidad.

Gutiérrez, R. (1978). Una nueva propuesta: el renacimiento colonial. En M. Waisman (coord.). Documentos para una historia de la arquitectura argentina (pp. 151154). Buenos Aires, Ediciones Summa.

(45) Lorente, Sánchez Giménez y CABAÑas Bravo (2009). 
(2002). Héctor Velarde. Lima: Epígrafe Editores.

- y Tartarini, J. (1997). El Banco de Boston. La Casa Central en la Argentina, 1917-1997. Buenos Aires: Fundación Banco de Boston.

Gutiérrez Viñuales, R. (2000). El 98 y la "reconquista espiritual" de América a través de la pintura. La influencia de Ignacio Zuloaga en la Argentina. En VII Congreso Internacional de Historia de América (AEA) (pp. 396-412). Las Palmas de Gran Canaria: Cabildo Insular.

- (2001). «Hermen Anglada Camarasa y Mallorca. Su significación para el arte iberoamericano. En X Jornadas de Historia del Arte «El arte español del siglo XX. Su perspectiva al final del milenio» (pp. 189-203). Madrid: Instituto de Investigaciones «Diego Velázquez», CSIC.

- (2003a). El Hispanismo como factor de mestizaje estético en el arte americano (1900-1930). En V. Mínguez (coord.). Iberoamérica Mestiza. Encuentro de pueblos y culturas (pp. 167-185). Madrid: Sociedad Estatal para la Acción Cultural Exterior-SEACEX.

- (2003b). La pintura argentina (1900-1930). Identidad Nacional e Hispanismo. Granada: Universidad, 2003.

(2004). Monumento conmemorativo y espacio público en Iberoamérica. Madrid: Cátedra.

- (2006). «El orientalismo en el imaginario artístico y urbano de Iberoamérica. Exotismo, fascinación e identidad». En J. A. González Alcantud (ed.). El orientalismo desde el sur (pp. 231-259). Sevilla: Anthropos.

- (2008). La Alhambra viajera. Rutas americanas de una obsesión romántica. En J. A. González Alcantud y A. Akmir (coords.). La Alhambra: lugar de la memoria y el diálogo (pp. 95-122). Granada: Comares Editorial.

- (2010). Arte y orientalismo en Iberoamérica. De la fantasía árabe a la edad del encantamiento. En J. A. González Alcantud (ed.). La invención del estilo hispanomarroquí. Presente y futuros del pasado (pp. 285-307). Rubí (Barcelona): Anthropos.

- (2014a). Arquitectura de raíces hispanas: entre los "estilos californianos" y el neocolonial (1880-1940). En M. A. Sorroche Cuerva (coord.). Baja California. Herencia, memoria e identidad patrimonial (pp. 281-307). Granada: Universidad de Granada-Editorial Atrio.

- (2014b). Libros argentinos. Ilustración y modernidad, 1910-1936. Buenos Aires: CEDODAL.

(2014c). Modernidad rioplatense. Libros ilustrados uruguayos en una era de transformaciones artísticas (1920-1934). Temas de la Academia, 73-84.

Hudson, A. (2000). The magic of Balboa Park. La Jolla: PhotoSecrets Publishing.

Ingle, M. (1984). Mayan revival style. Art Deco Mayan Fantasy. Salt Lake City: Peregrine Smith Books.

Keaton, D. y Waldie, D. J. (2007). California romántica. New York: Rizzoli.

Kuon Arce, E., Gutiérrez Viñuales, R., Gutiérrez, R. y Viñuales, G. (2009). CuzcoBuenos Aires. Ruta de intelectualidad americana (1900-1950). Lima: Universidad San Martín de Porres, Fondo Editorial. 
Laroche, W. E. (1992). Pintores uruguayos en España, 1900-1930. Montevideo: Galería de la Matriz.

Lemos, C. A. C. (1994). El estilo que nunca existió. En A. Amaral (comp.). Arquitectura Neocolonial. América Latina, Caribe, Estados Unidos (pp. 147-164). São Paulo: Fondo de Cultura Económica.

Lorente, J. P. Sánchez Giménez, S. y Cabañas Bravo, M. (coords.) (2009). Vae Victis! : Los artistas del exilio y sus museos. Gijón: Trea.

Lladó i Pol, F. (2006). Pintores argentinos en Mallorca (1900-1936). Palma de Mallorca: Lleonard Muntaner Editor.

Magalhães, F. (coord.) (1999). II Bienal Mercosul. Picasso, cubismo e América Latina. Porto Alegre: Fundação Bienal de Artes Visuais do Mercosul.

McMillian, E. (1996). Casa California. Spanish style houses from Santa Barbara to San Clemente. New York: Rizzoli.

- (2002). California Colonial. The Spanish and Rancho Revival Styles. Atglen: Schiffer Publishing.

Merino de Cáceres, J. M. y Martínez Ruiz, M. J. (2012). La destrucción del patrimonio artístico español.W.R. Hearst: «el gran acaparador». Madrid: Ediciones Cátedra.

Miralles, F. y Sanjuán, Ch. (2003). Anglada-Camarasa y Argentina. Sabadell: Editorial Ausa.

Pérez Sánchez, A. (1999). Pintura española en Chile. Valencia: Consorcio de Museos de la Comunitat Valenciana.

- (2000). Pintura española en el Museo Nacional de San Carlos de México. Valencia: Generalitat Valenciana.

Piñera Ramírez, D. , y Bejarano Suárez, A. S. (2011). Expresiones arquitectónicas compartidas en la frontera de Baja California y California. Culturales, VII (14), 159-184.

Raquejo, T. (1989). El palacio encantado. La Alhambra en el arte británico. Madrid: Taurus.

Ribeiro dos Santos, R. y Gutiérrez Viñuales, R. (2012). Arte latinoamericano en España, del 92 al momento actual. Vías de actuación y proyecciones. En R. López Guzmán (coord.). Andalucía y América (pp. 173-190). Baeza: Universidad Internacional de Andalucía.

Santa Ana Álvarez Osorio, F. de (dir.) (1995). Los salones Artal. Madrid: Ministerio de Cultura y Fundación Central Hispano.

Spanish homes of California (1925). Long Beach: Roy A. Hilton C. , Publishers.

Teixeira Leite, J. R. (1996). Pintores espanhóis no Brasil. São Paulo: Espaço Cultural Sérgio Barcellos.

Torre, S. (1994). En busca de una identidad regional: evolución de los estilos misionero y neocolonial hispano en California entre 1880 y 1930. En A. Amaral (comp.). Arquitectura Neocolonial. América Latina, Caribe, Estados Unidos (pp. 47-60). São Paulo: Fondo de Cultura Económica.

Vivoni Farage, E. y Álvarez Curbelo, S. (eds.) (1998). Hispanofilia. Arquitectura y vida en Puerto Rico. San Juan: Editorial de la Universidad de Puerto Rico.

Zamorano Pérez, P. E. (1994). El pintor F. Álvarez de Sotomayor y su huella en América. A Coruña: Universidade da Coruña, Servicio de Publicacións. 